\title{
Knowledge, Attitude and Practice of Dentists Prescribing Antibiotics in Periodontal diseases in Dental Colleges of Karachi
}

\author{
Usman Rahman ${ }^{1}$ \\ BDS \\ Ashar Nizamuddin Jamelle ${ }^{2}$ \\ BDS, M Clin Dent \\ Yousuf Ansari ${ }^{3}$ \\ BDS \\ Noman Nasrullah ${ }^{4}$ \\ BDS \\ Gulrukh Askary ${ }^{5}$ \\ BDS, MSc \\ Hamid Baig $^{6}$ \\ BDS, MFDS
}

OBJECTIVE: The objective of this study was to assess the Practice of dental practitioners with reference to the prescription of antibiotics in patients with periodontitis.

METHODOLOGY: A self-administered, structured questionnaire was distributed to Three hundred and Fifty subjects including dental students of final year BDS, house officer, and lecturers. The questionnaire inquired about the prescription of antibiotics in different types of periodontal diseases and the type of severity. Frequencies and percentages were calculated and the data was analyzed using SPSS software version 20.

RESULTS: For periodontal abscess and gingivitis respectively, $95.2 \%$ and $99.1 \%$ of the participants, reported they will not prescribe antibiotics. While, $44.8 \%$ of the participants reported they give antibiotics to patients with Necrotizing Ulcerative Periodontitis, 50.9\% preferred the use of antibiotics in patients with Aggressive Periodontitis while 57\% said they will give antibiotics in chronic periodontitis. Amoxicillin (53\%) was the preferred drug for periodontal cases followed by Augmentin $(34.2 \%)$.

CONCLUSION:There is a wide prescription of antibiotics by dental practitioners, without any scientific evidence to justify the use of anitbiotics in periodontal diseases cases.

KEYWORDS: Antibiotics, Over prescription, Resistance, Periodontal disease.

HOW TO CITE: Rahman U, Jamelle AN, Ansari Y, Nasrullah N, Askary G, Baig H . Knowledge, attitude and practice of dentists prescribing antibiotics in periodontal diseases in dental colleges of Karachi. J Pak Dent Assoc 2020;29(3):144-150.

DOI: https://doi.org/10.25301/JPDA.293.144

Received: 11 October 2019, Accepted: 08 June 2020

\section{INTRODUCTION}

I nfection in our body can spread and be severe and sometimes, the number of harmful bacteria is excessive, and the immune system cannot fight them all. Antibiotics are useful in this scenario. ${ }^{1,2}$

Antibiotics, also known as antibacterials, are medications through their selected action can destroy or slow down the growth of bacteria. ${ }^{1,2} \mathrm{~A}$ massive improvement in resolving infectious diseases has occurred since the introduction of antibiotics. Over the years, it has become a common practice

1. MDS Resident, Department of Periodontology, Fatima Jinnah Dental College.

2. Assistant Professor, Department of Periodontology, Fatima Jinnah Dental College.

3. Demonstrator, Department of Oral Medicine, Fatima Jinnah Dental College.

4. Demonstrator, Department of Periodontology, Fatima Jinnah Dental College.

5. Assistant Professor, Department of Oral Pathology, Fatima Jinnah Dental College.

6. Assistant Professor, Department of Oral Surgery, Fatima Jinnah Dental College.

Corresponding author: "Dr. Usman Rahman" < usmanrahman20014@ gmail.com > among dental professionals to prescribe antibiotic ther apy for the treatment of various dental infections and as prophylactic protocols. ${ }^{1,2}$

The first antibiotic was penicillin. Penicillin-based antibiotics, such as ampicillin, amoxicillin, and penicillin G, are still available to treat a variety of infections and are commonly used against bacteria in periodontal disease. There are different types of antibiotics, which work in one of two ways. A bactericidal antibiotic, such as penicillin, kills the bacteria. These drugs usually interfere with either the formation of the bacterial cell wall or its cell contents. Bacteriostatic stops bacteria from multiplying. ${ }^{1,3}$ In the practice of antibiotic prescription, as per international guidelines, certain factors like age of the patient, renal and hepatic function, drug allergies, pregnancy, systemic involvement (fever, malaise or lymph node) impaired host defense and local factors like presence of pus, necrotic 
material should be taken into consideration while making a decision for selecting the appropriate antibiotic. ${ }^{4}$

However, antibiotics, highly effective they may be against numerous deadly diseases, can be very easily misused and mismanaged which can have adverse consequences on the health of the human. Some medical professionals have concerns that people are overusing antibiotics. ${ }^{2,5}$ They also believe that this overuse contributes toward the growing number of bacterial infections that are becoming resistant to antibacterial medications. ${ }^{5}$ This concern is shared in the dentistry field. ${ }^{6}$

A study conducted in Canada, showed antibiotics prescribed by dentists had increased from $6.7 \%$ to $11 \%$ from the year 1996 to $2013 .{ }^{7}$ Further studies show that dentists have been prescribing 7 to $11 \%$ of all common antibiotics such as $\beta$-lactams, macrolides, tetracycline, clindamycin, metronidazole. ${ }^{8}$

For more than twenty years, dental surgeons and microbiologist have taken the support of periodontal antibiotic therapy strongly in conjunction with conventional mechanical debridement for treatment and management of periodontal disease. ${ }^{4}$ It is important to identify the causative pathogens and arrive at correct diagnosis in order for antibiotic periodontal therapy to be effective. A variety of antibiotics is available to treat various periodontal infections, but it is difficult to decide what drug will provide maximum benefits to the patients and will have little or no adverse effects. ${ }^{9}$

Lack of awareness and knowledge of the various antibiotics and their workings on the dentists' behalf has in many cases, allowed over prescription of antibiotics which then would be viewed as an overuse of the antibiotic medicine. This liberal use of antibiotics may lead to resistance to many microorganisms in humans and their environment. ${ }^{2,6}$ Few microbial species have completely become resistant towards the antibiotics in which methicillin - resistant staphylococcus aureus is a well-known example. Studies suggest that the prescription of systemic antibiotics should be restricted because almost all the dental and periodontal infections can be treated by clinical procedures and plaque control techniques. ${ }^{8}$ Numerous reports have been made about the unnecessary use of antibiotics by the dentist and the effect they have on antibiotic resistance including disturbance of protective commensal bacteria which is a problem being faced worldwide. ${ }^{6,10}$

According to the Centers for Disease Control (CDC), antibiotic use appears to be higher in some regions, such as the Southeast Asia. ${ }^{2}$ The variable of the amount of use of antibiotics varies from region to region, depending on a lot of factors namely; expertise, knowledge of antibiotics, freedom and permission to prescribe antibiotics, availability of the medicine in the area etc. ${ }^{2}$ Therefore, this study is to accurately assess the amount of usage of antibiotics and knowledge of dental practitioners with reference to the prescription of antibiotics in patients with periodontitis in dental colleges in Karachi, Pakistan. By doing this cross-sectional survey, gaps in the knowledge of the dental professional with reference to the prescription of antibiotics can be identified. The information can serve as a basis for further creation of educational measures that emphasize the need of antibiotic indications in periodontal therapy. A proper, controlled prescription of the antibiotics will then eliminate consequential risks of antibiotic resistance and hence, have a more positive impact on the health of the individual and the community overall.

\section{METHODOLOGY}

This cross-sectional study was conducted by using structured questionnaires with multiple-choice questions that allowed completion of responses. The study was conducted from January 2019 to May 2019, amongst four different teaching institutes of Karachi, Pakistan, that hold a lot of popularity for treatment of dental patients in Karachi. They were namely Liaqat College of Medicine and Dentistry, Dow University of Health Sciences, Altamash Institute of Dental Medicine, and Fatima Jinnah Dental College. Three hundred and fifty subjects took part in the study which comprised of dental students of final year BDS, house officers, and lecturers. 1st year, 2nd year and 3rd-year dental students were excluded from the group as only seasoned dental professionals who have completed the study of periodontology and have sufficient exposure of clinical practice sufficed for the study. Sample size was calculated using previous studies in which dentists who performed periodontal treatment in private clinics or in public service voluntarily participated in the study ${ }^{18}$ were included.

The questionnaire was distributed to the subjects and they filled in their responses. Before the study, the questionnaire was pilot tested on 5 dentists not involved in this project. Participants gave a written informed consent prior to the study and since there was no human intervention, exemption was taken from the ethical review board (DEC-2018-PER01). The questionnaire consisted of twenty close-ended questions. The questionnaire was based on the prescription of antibiotics in different types of periodontal diseases and the type of their severity. Examples of periodontal cases were presented and multiple choices were given on its treatment and whether antibiotic therapy was used as a solution to that case and which antibiotic. Questions focused on whether antibiotics were used as a treatment for gingivitis and periodontitis, as a sole therapy or in conjunction with mechanical therapy for periodontal disease, its use in root 
planning and perio surgical cases and whether they prescribed antibiotics as adjunct to periodontal surgery.

It also investigated their knowledge of prescribing antibiotics in systemic conditions such as diabetes, kidney disease, organ transplant, prosthetics, thrombocytopenia, asthmatic and cardiac diseases. Subjects' understanding of antibiotic therapy was tested in terms of effectiveness of types of antibiotics in periodontal cases either bacteriostatic, bactericidal or a combination of the two and the duration of antibiotic treatment in various examples of periodontal cases. Periodontal sampling for culture and sensitivity before prescribing antibiotics and the use of combination antibiotic therapy was looked into. The data was collected and SPSS software version 20 was used for the analysis of the quantitative data.

The data was presented in terms of the frequency of the prescription of the antibiotics commonly used in periodontal therapy, namely Amoxicillin, Tetracycline, Augmentin, and Metronidazole along with the frequency of the prescribing party who prescribed which antibiotic. The chosen antibiotics that have been presented in the data was concluded upon the results from the questionnaire that questioned which antibiotics were most commonly prescribed in periodontal therapy and how they were used. The sample were divided into three groups; undergraduates, house officers and demonstrators. Data was also presented in the frequency of the prescribing parties in prescribing antibiotics in systemic conditions namely diabetes, kidney disease, organ transplant, prosthetics, thrombocytopenia, asthmatic and cardiac diseases and the frequency of antibiotics usage in various cases of periodontal cases..

\section{RESULTS}

There were $330(94.2 \%)$ professionals who responded to the survey out of 350 . Mean age of the participants was $25 \pm 2$ years out of which 116 participants $(35.2 \%)$ were male and $214(64.8 \%)$ were females. Out of 330 respondents, $84(25.5 \%)$ were undergraduates, $131(39.7 \%)$ were House officers and $115(34.8 \%)$ were graduates having more than one year of experience.

When inquired about the indication of antibiotics in different types of periodontal disease 327 (99.1\%) did not prescribe any antibiotics for gingivitis. In chronic Periodontitis $188(57 \%)$ participants stated that they prescribe antibiotics, which out of this, $78 \%$ said they only prescribe antibiotics in severe chronic periodontitis cases while $20.5 \%$ said they give antibiotics in moderate and severe cases. The remaining $1.5 \%$ participants said they prescribe antibiotics in all forms of chronic periodontitis i.e. mild, moderate and severe.

In periodontal abscess 314 (95.2\%) participants said they do not prescribe antibiotics. Patients with Necrotizing Ulcerative Periodontitis 148 (44.8\%) participants said they give antibiotics, 168 (50.9\%) said they prescribed the use of antibiotics in patients with Aggressive Periodontitis while $177(53.6 \%)$ supported the prescription of antibiotics in patients suffering from Chronic Periodontitis associated with systemic diseases. Rest of the $50 \%$ of the participants said they do not give an antibiotic in Necrotizing Ulcerative Periodontitis, Aggressive Periodontitis and Chronic Periodontitis patients with systemic disease. Results have been summarized in Table 3 .

In healthy patients with no allergies, 175 (53\%) participants said they prescribe amoxicillin in most of the periodontal cases followed by Augmentin 113 (34.2\%). (Table 1)

In combination therapies, 134 (37.6\%) participants revealed they prescribe Augmentin and metronidazole as a combination therapy for periodontal cases while 118 (35.8\%) said they prescribe amoxicillin-metronidazole as a combination therapy in periodontal cases. The rest prescribed Cefadroxil- metronidazole combination therapy. An overview of the results of the antibiotics usually prescribed is in Table 1.

When inquired about the practice of mono-therapy or combination therapy, $269(81.5 \%)$ participants used combination therapy for moderate to severe Periodontitis

Table 1: Antibiotic prescription in various periodontal diseases

\begin{tabular}{|l|c|c|c|c|c|c|}
\hline & Gingivitis & $\begin{array}{c}\text { Chronic } \\
\text { Periodontitis } \\
(\%)\end{array}$ & $\begin{array}{c}\text { Necrotizing } \\
\text { Ulcerative } \\
\text { Periodontitis } \\
(\%)\end{array}$ & $\begin{array}{c}\text { Aggressive } \\
\text { Periodontitis } \\
(\%)\end{array}$ & $\begin{array}{c}\text { Periodontal } \\
\text { Abscess } \\
(\%)\end{array}$ & $\begin{array}{c}\text { Chronic } \\
\text { Periodontitis With } \\
\text { Systemic Condition } \\
(\%)\end{array}$ \\
\hline Yes & 0.9 & 57 & 44.8 & 50.9 & 4.8 & 53.6 \\
\hline No & 99.1 & 43 & 55.2 & 49.1 & 95.2 & 46.4 \\
\hline Total & 100 & 100 & 100 & 100 & 100 & 100 \\
\hline
\end{tabular}

Table 2: Frequent antibiotic Prescriptions

\begin{tabular}{|c|c|c|}
\hline & Frequency & Percent \\
\hline Amoxicillin & 175 & 53.0 \\
\hline Tetracycline & 23 & 7.0 \\
\hline Augmentin & 113 & 34.2 \\
\hline Metronidazole & 19 & 5.8 \\
\hline Total & 330 & 100.0 \\
\hline
\end{tabular}

cases, whereas, $237(71.8 \%)$ said they prescribe antibiotics in adjunct with Scaling and Root Planning. Fifty two percent of the participants stated that they do not consider prescribing antibiotics as prophylaxis before periodontal therapy in subacute bacterial endocarditis, while $47 \%$ confirmed that they do prescribe antibiotics as prophylaxis before periodontal 
Rahman U/ Jamelle AN/ Ansari Y/

Nasrullah N/ Askary G/ Baig H

Figure 1

Do you Prescribe antibiotic as a prophylaxis before Periodontal therapy in subacute bacterial endocarditis

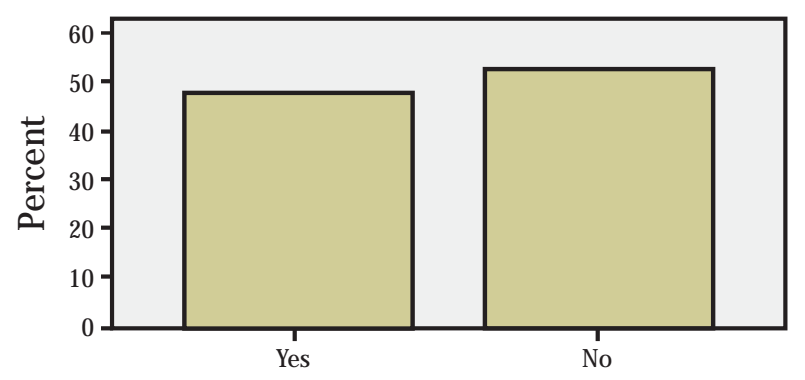

Table 3

\begin{tabular}{|l|c|c|c|}
\hline Questions & $\begin{array}{c}\text { YES } \\
(\%)\end{array}$ & $\begin{array}{c}\text { NO } \\
(\%)\end{array}$ & $\begin{array}{c}\text { TOTAL } \\
(\%)\end{array}$ \\
\hline $\begin{array}{l}\text { Do you Prescribe antibiotics as a prophylaxes before Periodontal } \\
\text { therapy in sub-acute bacterial endocarditis }\end{array}$ & 47.3 & 52.7 & 100 \\
\hline $\begin{array}{l}\text { do you take systemic consideration into account before prescribing } \\
\text { Antibiotics }\end{array}$ & 82.7 & 17.3 & 100 \\
\hline $\begin{array}{l}\text { Do you Prescribe antibiotics after periodontal surgeries for access } \\
\text { and Root Planning }\end{array}$ & 70.3 & 29.7 & 100 \\
\hline $\begin{array}{l}\text { Do you perform Periodontal sampling for culture and sensitivity } \\
\text { before prescribing antibiotics }\end{array}$ & 7 & 93 & 100 \\
\hline $\begin{array}{l}\text { Doyou take proper medical and drug history before prescribing } \\
\text { antibiotics }\end{array}$ & 65.5 & 34.5 & 100 \\
\hline $\begin{array}{l}\text { Do you prescribe antibiotic for Fibrous Gingival Enlargement } \\
\text { (Hyperplasia) }\end{array}$ & 20.6 & 79.4 & 100 \\
\hline
\end{tabular}

Table 1: Antibiotic prescription by subjects

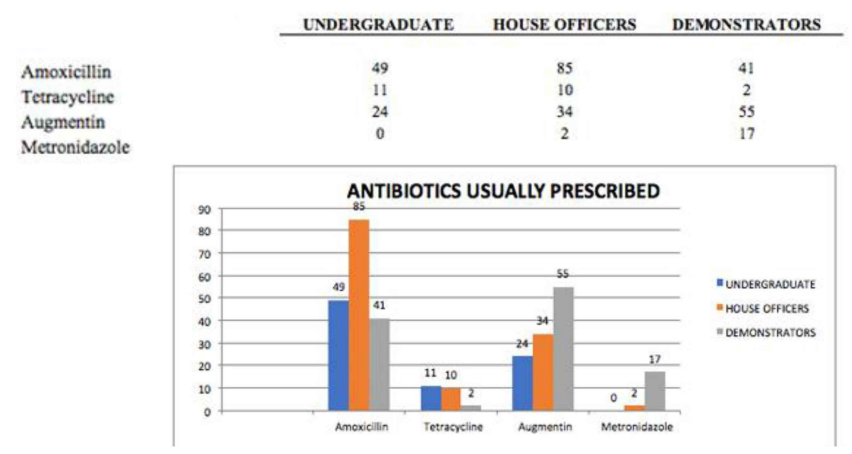

Table 2: Prescription in system conditions

\begin{tabular}{lccc} 
& UNDERGRADUATE & HOUSE OFFICERS & DEMONSTRATORS \\
\cline { 2 - 4 } DIABETES & 65 & 84 & 79 \\
KIDNEY DISEASE & 50 & 91 & 50 \\
ORGAN TRANSPLANT & 1 & 2 & 13 \\
PROSTHETICS & 33 & 41 & 66 \\
ASTHAMATIC & 0 & 0 & 0 \\
THROMBOCYTOPENIA & 5 & 24 & 12
\end{tabular}

therapy in sub-acute bacterial endocarditis. More than eighty percent $(82.7 \%)$ of the participants took systemic diseases into consideration before prescribing antibiotics while the remaining $17.3 \%$ did not. For open flap debridement and root planning, $70.3 \%$ of the participants prescribe antibiotics after periodontal surgeries.

In culture and sensitivity tests, $93 \%$ of the students did not perform periodontal sampling for culture and sensitivity
Practice of dentists prescribing antibiotics in periodontal diseases in dental colleges of Karachi

Table 3: Gingival and Periodontal disease precsription

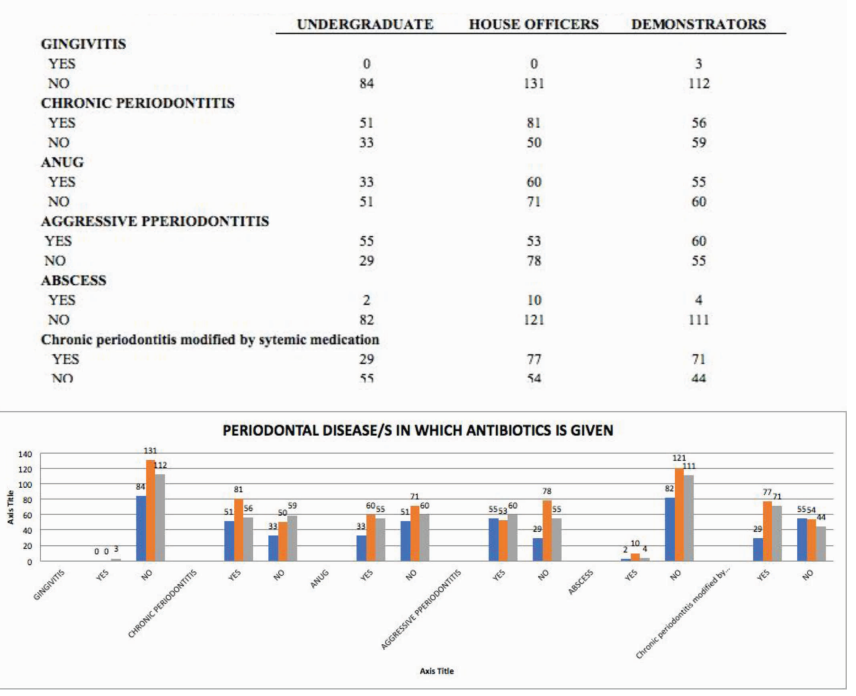

before the prescription of antibiotics, In this practice, house officers were $76 \%$ and demonstrators $30 \%$. More than a third $(34.5 \%)$ of the participants did not take proper medical and drug history while $65.5 \%$ took a detailed history before antibiotic prescription.

When inquired about prescription of antibiotics in gingival disease $79.4 \%$ of the students, $83.5 \%$ house officers, and 92.5\%. demonstrators did not prescribe antibiotics for Fibrous Gingival Enlargement (Hyperplasia). When asked about supportive use of antibiotics, in conjunction with periodontal therapeutic procedures, $67.9 \%$ of the participants prescribed antibiotics before and after scaling and Root Planing procedures while $15.5 \%$ said they only prescribe antibiotics before the procedures and $16.7 \%$ said they prescribe antibiotics after the periodontal procedure. Around $82.7 \%$ of participants of our study agreed that it is important to take systemic consideration into account to prescribe a suitable antibiotic. Over $3 / 4(76 \%)$ of our participants prescribed antibiotics in periodontal patients with diabetes mellitus as shown in Table 2.

Regarding the effectiveness of different antibiotics on various periodontal diseases, $186(56.4 \%)$ participants responded with sole bactericidal therapy whereas $31.8 \%$ chose bacteriostatic and bactericidal combination therapy as most effective against aggressive periodontitis. When asked about the duration for use of antibiotic prescription during treatment of Chronic Periodontitis 69.4\% said they prescribe antibiotics for 5 days, whereas for aggressive periodontitis $61.2 \%$ considered 1 week effective in the elimination of most periodontal pathogens.

\section{DISCUSSION}

Our study was a survey conducted in the teaching 
institutes specifically chosen as they are the root of medical and dental practitioners where undue prescription of antibiotics can be easily controlled. This study was conducted to accurately assess the rate of antibiotic prescription amongst dental professionals and to evaluate the prescription habits evaluating the various factors behind the reason for prescription of antibiotics. Current study reveals an accurate assessment of the frequency of usage of antibiotics by dentists in dental colleges of Pakistan by looking at the types of periodontal cases antibiotics are prescribed in, and which antibiotics are most commonly prescribed keeping in mind the subject's knowledge of its type and effectiveness in treatment. The data fluctuates between each prescribing party all handling the same case, and reveals their knowledge and practice of antibiotic therapy. The data revealed serves as a basis on how to improve the usage of antibiotic therapy and to make it more controlled and effective Monitoring and regulation of education providers, institutes, their teaching skills and the awareness of the teachers educating at these institutes regarding hazards of prescribing antibiotics unnecessarily will help solve the problem. The study used the prescription practices as a proxy measure for their knowledge (a hidden objective) but did not test the knowledge except for one question on importance of prescribing anitbiotics for systemic diseases.

In our study, a great number of professionals (99.1\%) reported that they did not prescribe antibiotics in patients with gingivitis. When we compared our study with Ismail et al's study we discovered that $50 \%$ of their participants said they do not prescribe antibiotics in gingivitis. ${ }^{11}$ The participants included house officers and faculty members of two dental colleges in Lahore. The reason behind this over prescription as suggested in this study was their inadequate knowledge of existing evidence of antibiotic use in dentistry. The study showed that participants in dental colleges of Karachi may have more awareness and adequate knowledge of gingivitis and the contradictory use of antibiotics in gingivitis cases.

Almost $70 \%$ of the respondents of our study said that they prescribed antibiotics as an adjunct to scaling and root planning. On the other hand, a study conducted by Yousufi et al reported that $45.2 \%$ of their respondents prescribed antibiotics as an adjunct to mechanical therapy. ${ }^{12}$

Participants (54.3\%) supported the use of post-operative antibiotics post periodontal procedures like flap surgeries. Little scientific evidence is present to support the use of antibiotics following periodontal flap surgery. Lack of awareness of evidence-based practice with regards to systemic antibiotic therapy may also be a reason.

Around $82.7 \%$ of participants of our study agreed that it is important to take systemic consideration into account and only then prescribe a suitable antibiotic. When specifically enquired about prescription of antibiotics in periodontal patients with diabetes mellitus, $69 \%$ of our participants agreed to give antibiotics in diabetic patients.

According to our study, $95.2 \%$ of the participants chose not to prescribe antibiotics in periodontal abscess patients and that abscesses should be treated through incision and drainage and mechanical debridement of the affected area. In a study carried out by Ismail et al it was found that $58 \%$ of their participants prefer giving antibiotics as an adjunct to mechanical debridement in periodontal abscess patients while $26 \%$ suggested no antibiotic prescription in patients with periodontal abscess. ${ }^{11}$ The studies showed that antibiotic therapy in patients with periodontal abscesses is unnecessary, as procedures like incision and drainage along with mechanical debridement and irrigation is enough to eliminate the cause and to eradicate the pathogenic microorganisms from the affected areas. In support of this point of view, it is recommended that use of antibiotic therapy in periodontal abscesses only if it is accompanied by systemic manifestations. ${ }^{4}$

In relevance to treatment of diseases like ANUG our study reported that $44.8 \%$. participants were in favor of prescribing antibiotics whereas in a study by Monteiro et al it was found that $45 \%$ of their participants preferred prescribing antibiotics to patients with Necrotizing ulcerative periodontitis which is similar to our study. ${ }^{14}$ Many studies suggest that patients with moderate or severe Necrotizing ulcerative gingivitis or necrotizing ulcerative periodontitis (NUP), local lymphadenopathy and systemic involvement need to be treated with antibiotic therapy, the recommended antibiotics are amoxicillin, metronidazole, and a combination of amoxicillin and metronidazole. ${ }^{4}$

When inquired about prescribing antibiotics to patients with aggressive periodontitis, $50 \%$ of the participants of our study suggested that antibiotics should be given in whereas in a similar study carried out by Monteiro et al it was reported that $62 \%$ of their participants agreed to give antibiotics in patients with aggressive periodontitis. ${ }^{14}$ Some studies have suggested that Aggregatibacter Actinomycetemcomitans are the primary causative pathogen in Localized Aggressive Periodontitis. These microorganisms can be eradicated effectively by using metronidazole-amoxicillin combination therapy. ${ }^{4}$

A question on prescription of antibiotics in relevance with chronic periodontitis was asked from the participants of our study and $78 \%$ of the participant said they will only prescribe antibiotics in severe chronic periodontitis cases and $20.5 \%$ said they will give an antibiotic in moderate and severe cases whereas $1.5 \%$ of the participants said they will prescribe antibiotics in all forms of chronic periodontitis 
whether Mild, Moderate of severe. A similar question was asked from the participants of a study carried out by Monteiro et $\mathrm{al}^{14}$ and it was found that $87 \%$ of the professionals indicated use of antibiotic therapy for severe periodontitis, $38 \%$ for moderate and $6 \%$ for mild periodontitis, while another study conducted by Fahad et $\mathrm{al}^{11}$ found that $14 \%$ of their participants said they give antibiotics only, for chronic periodontitis while 59\% reported that they prescribed antibiotics as an adjunct to mechanical debridement, whereas $27 \%$ of the participants of the study said that they do not prescribe antibiotics.

Research based evidence suggests that in chronic periodontitis, antibiotic therapy is usually recommended for patients showing progressive periodontal breakdown even after conventional mechanical treatment and in patients not responding to periodontal therapy and patients with recurrent disease and this shows there was over prescription seen according to our results.

When asked about use of antibiotics in patients with chronic periodontitis in our study, 134 (37.6\%) participants said they prescribe Augmentin and metronidazole as a combination therapy for periodontal cases whereas 118 $(35.8 \%)$ said they prescribe amoxicillin-metronidazole as a combination therapy in periodontal cases.

Ongoing research and studies suggest that periodontal disease has a broad range of micro-organisms ; therefore, combination therapy of Antibiotics gives better results because they act on wider range of microorganisms as compared to single antibiotics. It also stops the bacterial resistance by utilizing agents with overlapping antimicrobial spectra.(4)A dual regimen of metronidazole and amoxicillin has been reported to be effective against Aggregatibacter actinomycetemcomitans and Porphyromonas gingivalis -associated periodontal infections. ${ }^{13}$ Strict regulation of antibiotic policies is needed or else they will lose effectiveness against microbes. ${ }^{4}$ Antimicrobial resistance is a worldwide problem preferentially affecting low- and middle-income countries and our study has tried to highlight the overuse in periodontology. Our study did not cover other colleges in Karachi which would be ideal to get a better overview.

\section{CONCLUSION}

Interpretation of results of this study help us conclude that the prescription of antibiotics is still used abundantly by dental practitioners, without following any proper protocol or standard guidelines. Majority of the periodontal diseases gingivitis, mild, moderate or even/ chronic periodontitis or dental abscess can be treated with non-surgical or surgical periodontal interventions without the need of Antibiotics
Even in necrotizing ulcerative periodontitis antibiotics should be only prescribed in severe cases. With evidence based and scientific literature strictly adhered to and followed over prescription in periodontal diseases can be avoided.

\section{RECOMMENDATION}

In Pakistan, there is no legal obligation to undertake any continuing professional development courses; therefore, dentists may be following outdated guidelines, have inadequate knowledge of the use of antibiotics in periodontal cases. Use of antibiotics should be strictly monitored in hospitals and clinics. Special teams should be formed to control the misuse of antibiotics especially in the dental sector where prescribing antibiotics in those cases where it is not necessary. A consistent framework of guidelines with regards to antibiotic prescription in dental cases should be provided to teachers and students in all dental institutions and made easily accessible and a mandatory part of the curriculum. The guidelines need to be regularly reviewed and updated with new supporting evidence of treatment of periodontal cases with antibiotic therapy. Further studies and research for antibiotic therapy in periodontal cases should be supported and encouraged as part of the curriculum to create more supporting evidence. Other than teaching institutes, these regularly updated proper guidelines should be provided to all the hospitals and private clinics and strict monitoring should be done. All these measures can help construe a more coherent and consistent practice amongst dentists in the use and management of antibiotic treatment in periodontal treatment.

\section{CONFLICT OF INTEREST}

None declared

\section{REFERENCES}

1. Davey P, Marwick CA, Scott CL, Charani E, Mcneil K, Brown E, et al. Interventions to improve antibiotic prescribing practices for hospital inpatients. Cochrane Database of Systematic Reviews. 2017. https://doi.org/10.1002/14651858.CD003543.pub4

2. Antibiotics: Uses, resistance, and side effects [Internet]. [cited 2020 Apr 22]. Available from: https://www.medicalnewstoday.com/ articles $/ 10278$

3. Gaynes R. The Discovery of Penicillin-New Insights after More Than 75 Years of Clinical Use. Emerg. Infect. Dis. 2017;23:849-53 Google Search [Internet]. [cited 2020 Apr 22].

https://doi.org/10.3201/eid2305.161556

4. Kapoor A, Malhotra R, Grover V, Grover D. Systemic antibiotic 
Rahman U/ Jamelle AN/ Ansari Y/

Nasrullah N/ Askary G/ Baig H

therapy in periodontics. Dent Res J (Isfahan). 2012;

https://doi.org/10.4103/1735-3327.104866

5. Llor C, Bjerrum L. Antimicrobial resistance: Risk associated with antibiotic overuse and initiatives to reduce the problem. Therapeutic Advances in Drug Safety. 2014.

https://doi.org/10.1177/2042098614554919

6. Cope AL, Chestnutt IG. Inappropriate prescribing of antibiotics in primary dental care: reasons and resolutions. Prim Dent J. 2014;3:3337.

https://doi.org/10.1308/205016814813877333

7. Marra F, George D, Chong M; Antibiotic prescribing by dentists has increased, why? J Am Dent Assoc. 2016;147:307-07 - Google Search [Internet]. [cited $2020 \mathrm{Apr} 22$ ].

https://doi.org/10.1016/j.adaj.2015.12.014

8. Dar-Odeh NS, Abu-Hammad OA, Al-Omiri MK, Khraisat AS, Shehabi AA. Antibiotic prescribing practices by dentists: a review. Ther Clin Risk Manag 2010 ;6:301-306 - Google Search [Internet]. [cited 2020 Apr 22].

https://doi.org/10.2147/TCRM.S9736

9. Soares GMS, Figueiredo LC, Faveri M, Cortelli SC, Duarte PM, Feres M. Mechanisms of action of systemic antibiotics used in
Practice of dentists prescribing antibiotics in periodontal diseases in dental colleges of Karachi

periodontal treatment and mechanisms of bacterial resistance to these drugs. J Applied Oral Sci. 2012.

https://doi.org/10.1590/S1678-77572012000300002

10. Ramasamy A. A review of use of antibiotics in dentistry and recommendations for rational antibiotic usage by dentists. Int Arab J Antimicrob Agents. 2014;4:1-15.

11. Ismail F, Qazia S, SajjadaA. Antibiotics prescription habits and knowledge of dentists in a lahore sample Pak Oral Dent J. 2018;38: 79-84 - Google Search [Internet]. [cited 2020 Apr 22].

12. Yousufi S, Israr $\mathrm{Y}$, Zaman $\mathrm{S}$ et al, Use of Antibiotics in Dental Teaching Hospitals of Peshawar, Pakistan: How Justified Are We? Int J Dent Oral Health. 2019;5:68-73 - Google Search [Internet]. [cited 2020 Apr 22].

13. Prakasam A, Elavarasu Ss, Natarajan R. Antibiotics in the management of aggressive periodontitis. J Pharm Bioallied Sci. 2012; https://doi.org/10.4103/0975-7406.100226

14. Monteiro AV , Ribeiro FV , Casarin RC. Evaluation of the use of systemic antimicrobial agents by professionals for the treatment of periodontal diseases. Braz J Oral Sci. 2013;12:285-291 - Google Search [Internet]. [cited 2020 Apr 22].

https://doi.org/10.1590/S1677-32252013000400003 